Mathematical Modelling and Analysis

Volume 20 Number 2, March 2015, 261-272

http://dx.doi.org/10.3846/13926292.2015.1021720

(c) Vilnius Gediminas Technical University, 2015
Publisher: Taylor\&Francis and VGTU

http://www.tandfonline.com/TMMA

ISSN: $1392-6292$

eISSN: $1648-3510$

\title{
Quantitative Approximation Properties for Iterates of Moment Operator
}

\section{Carlo Bardaro, Loris Faina and Ilaria Mantellini}

\author{
Department of Mathematics and Informatics, University of Perugia \\ Via Vanvitelli 1, 06123 Perugia, Italy \\ E-mail(corresp.): carlo.bardaro@unipg.it \\ E-mail: loris.faina@unipg.it \\ E-mail: ilaria.mantellini@unipg.it
}

Received June 23, 2014; revised January 20, 2015; published online March 15, 2015

\begin{abstract}
Here we state a quantitative approximation theorem by means of nets of certain modified Hadamard integrals, using iterates of moment type operators, for functions $f$ defined over the positive real semi-axis ]0, $+\infty$ [, having Mellin derivatives. The main tool is a suitable $K$-functional which is compatible with the structure of the multiplicative group $] 0,+\infty[$. Some numerical examples and graphical representations are illustrated.
\end{abstract}

Keywords: iterates of moment kernel, Mellin derivatives, generalized Hadamard integrals, $K$-functional.

AMS Subject Classification: 41A35; 41A25; 47G10.

\section{Introduction}

The theory of the so-called moment (or average) operators in approximation theory was first considered in the papers [4,31]. These operators have many applications to various fields of pure and applied mathematics: from Calculus of Variations (see $[2,28]$ ) to fractional calculus $[6,20]$ and stochastic processes $[14,18]$. In the frame of approximation theory by sequences or nets of moment operators we quote the papers $[3,13,27,29,30]$. Recently in $[6,7,8,9,10,11]$, the asymptotic behaviour was studied, obtaining Voronovskaya type formulae. In particular in [9], linear combinations of moment type operators are considered, which generate sequences of linear operators with a better order of pointwise and uniform approximation. In [11] special kinds of iterated moment operators were considered and it was remarked that linear combinations of iterated moment operators further improve the order of approximation.

In the present paper we point out the deep connections between of our iterated operators and the generalized Hadamard integrals, introduced firstly in [22], and widely used in Mellin analysis, especially in fractional calculus in 
Mellin transform setting (see e.g. $[5,17,23]$ ). This link enables us to obtain corresponding results concerning the approximation of a function $f$ defined over the positive real semi-axis ]0, $+\infty$ [, by means of nets of slightly modified generalized Hadamard operators. The main result is Theorem 2, which gives a quantitative estimate of the pointwise and uniform convergence in certain classes of differentiable functions in the Mellin sense (see $[5,16])$. We use here a suitable Peetre $K$-functional, and a technique introduced in [21]. The last section considers some suitable examples which illustrate the results by means of graphical representations and numerical calculations. These calculations point out that without certain global regularity assumptions on the function $f$ we cannot obtain the uniform convergence over $] 0,+\infty[$. This gives the main motivation for the assumptions of Theorems 1 and 2 .

\section{Preliminaries}

Let $\mathbb{R}^{+}$be the set of all positive real numbers endowed with the measure $\mu(E)=$ $\int_{E} \frac{d t}{t}$, where $E$ is any (Lebesgue) measurable set. We denote $\mathbb{R}_{0}^{+}=\mathbb{R}^{+} \cup\{0\}$, and by $\mathbb{N}, \mathbb{N}_{0}$ we denote the set of positive integers and non negative integers respectively. We will denote by $L^{p}(\mu), 1 \leq p<+\infty$ the Lebesgue spaces of $p$-integrable functions with respect to the measure $\mu$ and by $L^{\infty}(\mu)$ the space of all the essentially bounded functions. We will denote by $\|f\|_{p}$ and $\|f\|_{\infty}$, the corresponding norms.

For $m \in \mathbb{N}_{0}$ we denote by $C^{m}=C^{m}\left(\mathbb{R}^{+}\right)$the space of all functions $f: \mathbb{R}^{+} \rightarrow \mathbb{R}$ with continuous m-order derivative in $\mathbb{R}^{+}$. In particular $C^{0}$ will denote the space of all the continuous functions over $\mathbb{R}^{+}$. Moreover, we will say that $f \in C^{m}$ locally at the point $s \in \mathbb{R}^{+}$if there is a neighbourhood $U_{s}$ of the point $s$ such that $f$ is $(m-1)$-times continuously differentiable in $U_{s}$ and the derivative of order $m$ exists at the point $s$.

In the following we will say that a function $f$ is log-uniformly continuous in $\mathbb{R}^{+}$if for every $\varepsilon>0$ there exists $\eta>0$ such that $\left|f\left(s_{1}\right)-f\left(s_{2}\right)\right|<\varepsilon$ whenever $\left|\log s_{1}-\log s_{2}\right|<\eta$. This notion was firstly given, in an equivalent form, in [25, page 7], in terms of the dilation operator. Note that there are functions uniformly continuous in the usual sense but not in the log-sense and conversely. For example the function $f(u)=\sin u$ is obviously uniformly continuous but not in the $\log$-sense, while the function $g(u)=\sin (\log u)$ is $\log$-uniformly continuous but not in the usual sense. However, the two notions are equivalent on every bounded interval $[a, b]$ with $a>0$.

We denote by $B C^{0}$ the subspace of $C^{0}$ containing the log-uniformly continuous and bounded functions in $\mathbb{R}^{+}$and by $B C^{m}$ the space of all the functions such that the $k$-order derivatives, $k=1, \ldots m$, are in $B C^{0}$. We recall that the Mellin derivatives of $f$ are defined recursively in the following way $[5,16,25]$

$$
\Theta f(s)=s f^{\prime}(s), \quad \Theta^{m} f(s)=\Theta \circ \Theta^{m-1} f(s), \quad m \geq 2 .
$$

In particular, we have also the following Taylor formula of order $m$ for a function $f$ in $B C^{m}$ (see $\left.[10,25]\right)$, 


$$
\begin{aligned}
f(s t)= & f(s)+\Theta f(s) \log t+\frac{\Theta^{2} f(s)}{2 !} \log ^{2} t+\cdots+\frac{\Theta^{m} f(s)}{m !} \log ^{m} t \\
& +h_{s}(t) \log ^{m} t, \quad s, t>0,
\end{aligned}
$$

where $h_{s}(t) \rightarrow 0$ as $t \rightarrow 1$. If $f$ is bounded, we can assume that $h_{s}$ is also bounded.

\section{The Moment Operator and Its Iterates}

Let $K: \mathbb{R}^{+} \times \mathbb{R}^{+} \rightarrow \mathbb{R}_{0}^{+}$be the function defined by

$$
K(s, t)=\left(\frac{t}{s}\right) \chi_{] 0, s[}(t) .
$$

It is easy to see that $K$ is homogeneous of degree zero, i.e. $K(\lambda s, \lambda t)=K(s, t)$, for every $\lambda, s, t>0$, and

$$
\int_{0}^{+\infty} K(s, t) \frac{d t}{t}=1 .
$$

For every $j \in \mathbb{N}$ we define the logarithmic moment of order $j \in \mathbb{N}$ of $K$ as

$$
m_{j}(K):=\int_{0}^{+\infty} K(s, t) \log ^{j}\left(\frac{t}{s}\right) \frac{d t}{t}
$$

and, using the change of variable $z=t / s$, we have easily

$$
m_{j}(K)=\int_{0}^{1} \log ^{j} z d z=(-1)^{j} j !
$$

For the absolute logarithmic moment of order $j$, namely

$$
M_{j}(K):=\int_{0}^{+\infty}|K(s, t)|\left|\log ^{j}\left(\frac{t}{s}\right)\right| \frac{d t}{t}
$$

we have $M_{j}(K)=j$ !. The Mellin-Fejer kernel generated by $K$ is given by, for $w>0$,

$$
K_{w}(s, t)=w\left(\frac{t}{s}\right)^{w} \chi_{] 0, s[}(t) .
$$

The corresponding moment operator is given by

$$
\left(\mathcal{M}_{w} f\right)(s)=\int_{0}^{s} w\left(\frac{t}{s}\right)^{w} f(t) \frac{d t}{t}, \quad w>0 .
$$

We obtain that the corresponding logarithmic moments of order $j$ of the functions $K_{w}$ are

$$
m_{j}\left(K_{w}\right)=\frac{(-1)^{j} j !}{w^{j}}, \quad M_{j}\left(K_{w}\right)=\frac{j !}{w^{j}} .
$$


Given the function $K$ we define for every $n \in \mathbb{N}$ the iterated kernel of order $n$ of $K$ in the following way: for $n=2$,

$$
K^{2}(s, t)=\int_{0}^{+\infty} K(s, z) K(z, t) \frac{d z}{z},
$$

and for $n>2$,

$$
K^{n}(s, t)=\int_{0}^{+\infty} K(s, z) K^{n-1}(z, t) \frac{d z}{z} .
$$

It turns out that the iterated functions $K^{n}$ exist and for the corresponding Mellin-Fejer kernels we have the following result

Proposition 1. For $n=2,3, \ldots$, we have

$$
K_{w}^{n}(s, t)=\frac{w^{n}}{(n-1) !}\left(\frac{t}{s}\right)^{w} \log ^{n-1}\left(\frac{s}{t}\right) \chi_{] 0, s[}(t)
$$

with moments

$$
m_{j}\left(K_{w}^{n}\right)=\frac{(-1)^{j}}{w^{j}} \prod_{i=0}^{j-1}(n+i), \quad M_{j}\left(K_{w}^{n}\right)=\frac{1}{w^{j}} \prod_{i=0}^{j-1}(n+i) .
$$

Proof. For $n=2$ we have

$$
\begin{aligned}
K_{w}^{2}(s, t) & =\int_{0}^{+\infty} w^{2} z^{w} s^{-w} t^{w} z^{-w} \chi_{] 0, s[}(z) \chi_{] t,+\infty[}(z) \frac{d z}{z} \\
& =w^{2}\left(\frac{t}{s}\right)^{w} \chi_{] 0, s[}(t) \log \left(\frac{s}{t}\right)
\end{aligned}
$$

and the general case follows by an easy induction. The formulae for the moments follow using Proposition 7 in [11].

The iterated moment operator of order $n$ is defined as

$$
\left(\mathcal{M}_{w}^{n} f\right)(s)=\int_{0}^{+\infty} K_{w}^{n}(s, t) f(t) \frac{d t}{t}, \quad w>0
$$

for every $f$ for which the integral is well defined as a Lebesgue integral.

The above integrals are strictly related with the so-called Hadamard-type integrals, introduced for the first time in [22], and then used in the frame of Mellin analysis, (see e.g. $[5,17,23]$ ). Here we give a slightly different version of such integrals.

For $\alpha>0$ and $c>0$, we define the modified generalized Hadamard integral of order $\alpha$ of a function $f: \mathbb{R}^{+} \rightarrow \mathbb{R}$ as:

$$
\left(J_{0+, c}^{\alpha} f\right)(s)=\frac{c^{\alpha}}{\Gamma(\alpha)} \int_{0}^{s}\left(\frac{t}{s}\right)^{c}\left(\log \left(\frac{s}{t}\right)\right)^{\alpha-1} f(t) \frac{d t}{t} .
$$


As recently proved in [5], if $\alpha>1$, the domain of the operator $J_{0+, c}^{\alpha}$ is contained in the space

$$
X_{c, l o c}:=\left\{f: \mathbb{R}^{+} \rightarrow \mathbb{R}:(\cdot)^{c} f(\cdot) \in L_{l o c}^{1}\right\} .
$$

Moreover, it contains the space

$$
\widetilde{X}_{c, l o c}:=\left\{f \in X_{c, l o c}: \exists r \in\right] 0,1\left[\text {, such that } f(u)=\mathcal{O}\left(u^{-(r+c-1)}\right), u \rightarrow 0^{+}\right\} .
$$

As an immediate consequence of the definition of the modified Hadamard integrals, we have

$$
\left(\mathcal{M}_{w}^{n} f\right)(s)=\left(J_{0+, w}^{n} f\right)(s), \quad w>0 .
$$

\section{Asymptotic Behaviour of Generalized Hadamard Inte- grals via Moment Operators}

We are interested in the asymptotic behaviour of the generalized Hadamard integrals of order $n$ as $w \rightarrow+\infty$, using the above relation and the properties of the iterated moment operators. At first, as an immediate consequence of (3.1) and Corollary 4 in [11] we obtain

Theorem 1. Let $f \in L^{\infty}(\mu) \cap \bigcap_{w \geq 1} \widetilde{X}_{w, l o c}$ be fixed. Let $f \in C^{1}$ locally at $s \in \mathbb{R}^{+}$. Then we have

$$
\lim _{w \rightarrow+\infty} w\left[\left(J_{0+, w}^{n} f\right)(s)-f(s)\right]=-n \Theta f(s) .
$$

In particular,

$$
\left|w\left(J_{0+, w}^{n} f\right)(s)-f(s)\right|=\mathcal{O}\left(w^{-1}\right) \quad(w \rightarrow+\infty),
$$

where the $\mathcal{O}$-term depends on the point s. If $f \in B C^{1}$ then the estimate is uniform on $\mathbb{R}^{+}$.

Proof. The first assertion follows easily from (3.1) and Corollary 4 in [11]. For the second assertion, if $f \in B C^{1}$, then $|\Theta f(s)| \leq C$, for an absolute constant $C>0$, and so the $\mathcal{O}$-term is independent of $s$.

In the next section we will show by an example that the assumption $f \in$ $B C^{1}$ is essential for the uniform convergence over the whole positive real semiaxis.

Our aim is to obtain a quantitative version of Theorem 1 using a $K$ functional suitable for the Mellin setting.

In general, the (modified) Peetre $K$-functional is defined in the following way. Let $\left(X,\|\cdot\|_{X}\right)$ be a Banach space, $Y \subset X$ be a semi-normed subspace, with the seminorm $|\cdot|_{Y}$ such that $Y$ is complete under the norm $\|f\|_{Y}:=\|f\|_{X}+|f|_{Y}$. Then we put

$$
\mathcal{K}(f, t, X, Y):=\inf _{g \in Y}\left\{\|f-g\|_{X}+t|g|_{Y}\right\} .
$$


Taking $X=B C^{0}$ and $Y=B C^{1}$, our $K$-functional is defined by

$$
\mathcal{K}\left(f, \varepsilon, B C^{0}, B C^{1}\right) \equiv \mathcal{K}(f, \varepsilon):=\inf \left\{\|f-g\|_{\infty}+\varepsilon\|\Theta g\|_{\infty}: g \in B C^{1}\right\}
$$

for $f \in B C^{0}$ and $\varepsilon \geq 0$. By Corollary 1 in [12], it follows that this functional is well defined in the sense that $\lim _{\varepsilon \rightarrow 0} \mathcal{K}(f, \varepsilon)=0$. For a general treatment of $K$-functionals, see $[1,15,19,24,26]$. The intimate connections of a K-functional with approximation theory were developed in [15] and [19].

Associated with our $K$-functional, we introduce certain Lipschitz classes of functions, as follows. For $\alpha \in] 0,1]$, we set

$$
\operatorname{Lip}_{K}(\alpha)=\left\{f \in B C^{0}: \mathcal{K}(f, t)=\mathcal{O}\left(t^{\alpha}\right), t \rightarrow 0^{+}\right\} .
$$

In [12] the following estimate of the remainder $R_{m}(f ; s, t):=h_{s}(t) \log ^{m} t$ in the Taylor formula of order $m$ is proved, which extends a result proved in [21] for the classical Taylor formula in a slightly different setting.

Lemma 1. For $m \in \mathbb{N}$ let $f \in B C^{m}$ and $s, t \in \mathbb{R}^{+}$. Then we have

$$
\left|R_{m}(f ; s, t)\right| \leq \frac{2\left|\log ^{m} t\right|}{m !} \mathcal{K}\left(\Theta^{m} f, \frac{|\log t|}{2(m+1)}\right) .
$$

In particular, for $m=1$ we have, for functions $f \in B C^{1}$,

$$
\left|R_{1}(f, s, t)\right|=\left|h_{s}(t) \log t\right| \leq 2|\log t| \mathcal{K}\left(\Theta f, \frac{|\log t|}{4}\right) .
$$

We have the following

Theorem 2. Under the assumptions of Theorem 1, if $f \in B C^{1}$, we have the following estimate, for $w>0$,

$$
\left|w\left[\left(J_{0+, w}^{n} f\right)(s)-f(s)\right]+n \Theta f(s)\right| \leq 2 n \mathcal{K}\left(\Theta f, \frac{n+1}{4} \frac{1}{w}\right) .
$$

Proof. We start with the estimate of the difference

$$
J:=\left|\left(J_{0+, w}^{n} f\right)(s)-f(s)+\frac{n}{w} \Theta f(s)\right| .
$$

Using the Taylor formula of the first order, and taking into account that

$$
\int_{0}^{+\infty} K_{w}^{n}(s, t) \frac{d t}{t}=1
$$

for every $n \in \mathbb{N}$ and $s>0$, we have

$$
\begin{aligned}
\left(J_{0+, w}^{n} f\right)(s)-f(s)= & \Theta f(s) \int_{0}^{+\infty} K_{w}^{n}(s, t) \log \left(\frac{t}{s}\right) \frac{d t}{t} \\
& +\int_{0}^{+\infty} K_{w}^{n}(s, t) h\left(\frac{t}{s}\right) \log \left(\frac{t}{s}\right) \frac{d t}{t} .
\end{aligned}
$$


Therefore,

$$
\begin{aligned}
J \leq & \left|\int_{0}^{\infty} K_{w}^{n}(s, t) \log \left(\frac{t}{s}\right) \frac{d t}{t}+\frac{n}{w}\right||\Theta f(s)| \\
& +\int_{0}^{\infty}\left|K_{w}^{n}(s, t)\right|\left|R_{1}\left(f, s, \frac{t}{s}\right)\right| \frac{d t}{t}=J_{1}+J_{2} .
\end{aligned}
$$

For $J_{1}$ we have, by the notion of moments and Proposition 1

$$
J_{1}=\left|m_{1}\left(K_{w}^{n}\right)+\frac{n}{w}\right||\Theta f(s)|=0,
$$

while for $J_{2}$ using Lemma 1 and a simple change of variable,

$$
J_{2} \leq \frac{2 w^{n}}{(n-1) !} \int_{0}^{1} v^{w} \log ^{n-1}\left(\frac{1}{v}\right)|\log v| \mathcal{K}\left(\Theta f, \frac{|\log v|}{4}\right) \frac{d v}{v} .
$$

Now, let us consider an arbitrary function $g \in B C^{2}$. Then,

$$
\begin{aligned}
J_{2} & \leq \frac{2 w^{n}}{(n-1) !} \int_{0}^{1} v^{w} \log ^{n-1}\left(\frac{1}{v}\right)|\log v|\left(\|\Theta(f-g)\|_{\infty}+\frac{|\log v|}{4}\left\|\Theta^{2} g\right\|_{\infty}\right) \frac{d v}{v} \\
& =2 M_{1}\left(K_{w}^{n}\right)\|\Theta(f-g)\|_{\infty}+\frac{1}{2} M_{2}\left(K_{w}^{n}\right)\left\|\Theta^{2} g\right\|_{\infty} \\
& =\frac{2 n}{w}\left[\|\Theta(f-g)\|_{\infty}+\frac{1}{4} \frac{n+1}{w}\left\|\Theta^{2} g\right\|_{\infty}\right] .
\end{aligned}
$$

Taking now the infimum over all functions $g \in B C^{2}$, we get

$$
J_{2} \leq \frac{2 n}{w} \mathcal{K}\left(\Theta f, \frac{n+1}{4} \frac{1}{w}\right)
$$

Thus, the assertion follows.

As a consequence of Theorem 2, for functions $f \in B C^{2}$ we have the following direct estimate

Corollary 1. If $f \in B C^{2}$, we have

$$
\left|w\left[\left(J_{0+, w}^{n} f\right)(s)-f(s)\right]+n \Theta f(s)\right| \leq \frac{n(n+1)}{2} \frac{1}{w}\left\|\Theta^{2} f\right\|_{\infty} .
$$

Proof. If $f \in B C^{2}$, then we can write

$$
\mathcal{K}\left(\Theta f, \frac{(n+1)}{4 w}\right)=\inf _{g \in B C^{1}}\left\{\|\Theta f-g\|_{\infty}+\frac{(n+1)}{4 w}\|\Theta g\|_{\infty}\right\} \leq \frac{(n+1)}{4 w}\left\|\Theta^{2} f\right\|_{\infty},
$$

by choosing $g=\Theta f$. Therefore the assertion easily follows.

More generally, we have the following further consequence of Theorem 2, whose proof is clear.

Corollary 2. Let $f \in B C^{1}$ such that $\Theta f \in \operatorname{Lip}_{K}(1)$. Then

$$
\left|w\left[\left(J_{0+, w}^{n} f\right)(s)-f(s)\right]+n \Theta f(s)\right|=\mathcal{O}\left(w^{-1}\right), \quad w \rightarrow+\infty,
$$

where the $\mathcal{O}$-term is uniform with respect to $s$. 


\section{Examples and Some Numerical Evaluation}

We give two examples to show the asymptotic behaviour of the generalized Hadamard integrals (of the second order); the first involving the function $e^{s}$ (see Figure 1) and the second involving the function $\sin (\log s)$ (see Figure 2). Note that the function $f(s)=e^{s}$ does not belong to the space $B C^{1}$ since its Mellin derivative is not bounded over the whole positive real semi-axis. As we will show, we have a uniform convergence over the compact subsets of $\mathbb{R}^{+}$but we have not the uniform convergence over the positive semi-axis. The second function $f(x)=\sin (\log x)$ belongs to $B C^{2}$ since its Mellin derivative $\Theta^{2} f$ is given by $-\sin (\log x)$ and so $\left\|\Theta^{2} f\right\|_{\infty}=1$. The estimate of Corollary 1 , gives now

$$
\left|w\left[\left(J_{0+, w}^{n} f\right)(s)-f(s)\right]-n \cos (\log s)\right| \leq \frac{n(n+1)}{2} \frac{1}{w},
$$

which gives a uniform estimate of the asymptotic formula.

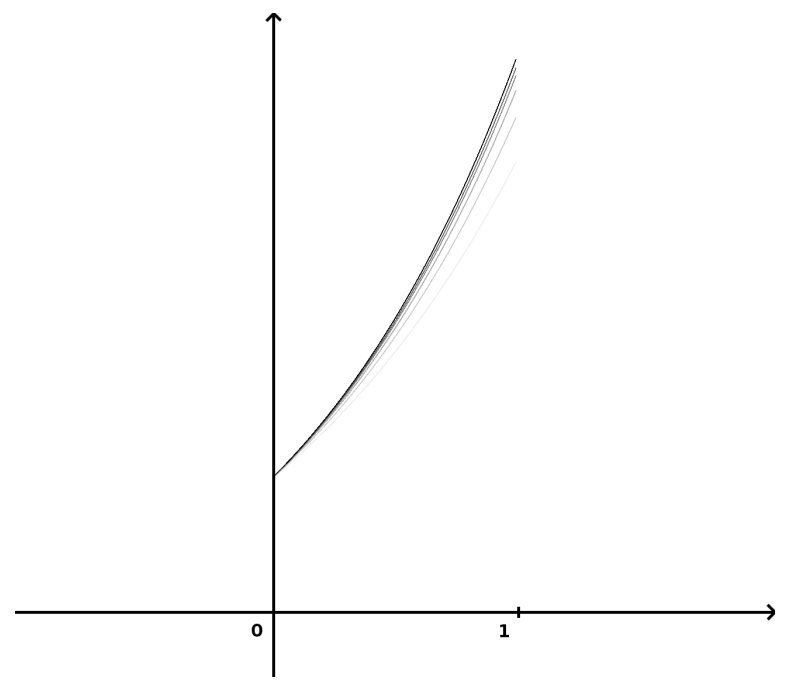

Figure 1. Uniform convergence of the modified generalized Hadamard integrals of order 2 of the function $e^{x}$ on the interval ]0,1]. As the grey level becomes darker the value of the parameter $\mathrm{w}$ increases of a factor 2 starting from 10. Seven approximations are shown, therefore from $w=10$ up to $w=640$.

The generalized Hadamard integrals $\left(J_{0^{+}, w}^{2} f\right)(s)$ are computed numerically using a modified trapezoidal rule with points, taking into account the singularity at the point zero; that is, the first interval of the integration is further divided into $10 * w$ points. We use this method in the spirit of a mere example; this is not the best method for integrating such integrals. Indeed, one of the Referees kindly suggested the use of a Gauss-Legendre quadrature rule. We report the results found by the Referee in the last column of each table. For the graphics of Figures 1 and 2 we took a discretization of 0.01 . Tables 1 and 2 report the values of the generalized Hadamard integrals $\left(J_{0^{+}, w}^{2} f\right)(s)$ evaluated 


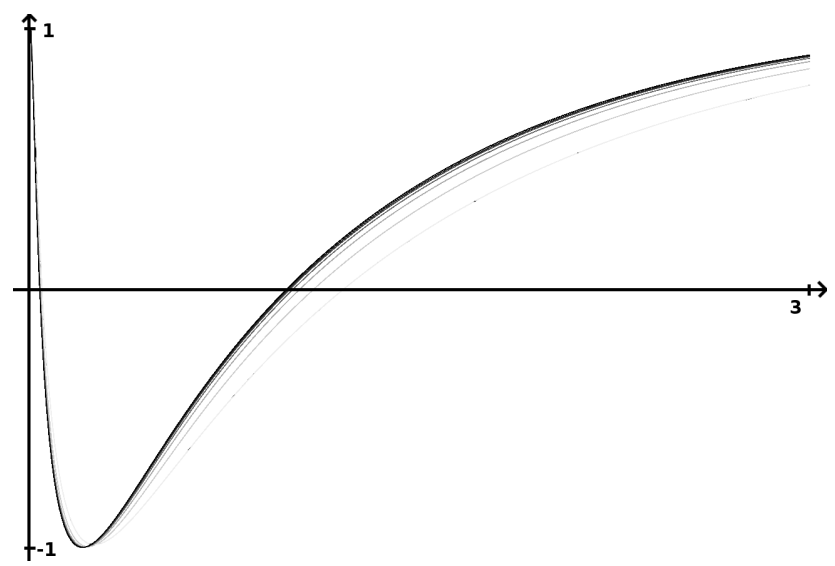

Figure 2. Uniform convergence of the modified generalized Hadamard integrals of order 2 of the function $\sin (\log x)$ on the interval ]0,3]. As the grey level becomes darker the value of the parameter $w$ increases of a factor 2 starting from 10. Seven approximations are shown, therefore from $w=10$ up to $w=640$.

Table 1. Hadamard integrals values for $f=e^{x}$ on $s=0.5$.

\begin{tabular}{rll}
\hline$w$ & $e^{0.5}-\left(J_{0^{+}, w}^{2} f\right)(0.5)$ & $\begin{array}{l}\text { Gauss-Legendre quadrature } \\
\text { rule with } 8192 \text { points }\end{array}$ \\
\hline 10 & 0.136285 & 0.1349117163 \\
20 & 0.075532 & 0.0741588938 \\
40 & 0.040405 & 0.0390320567 \\
80 & 0.021419 & 0.0200465051 \\
160 & 0.011535 & 0.0101617769 \\
320 & 0.006489 & 0.0051163015 \\
640 & 0.003940 & 0.0025671047 \\
1280 & 0.002659 & 0.0012858036 \\
2560 & 0.002016 & 0.0006434662 \\
5120 & 0.001695 & 0.0003218744 \\
10240 & 0.001534 & 0.0001609726 \\
20480 & 0.001453 & 0.0000804951 \\
40960 & 0.001413 & 0.0000402498 \\
81920 & 0.001393 & 0.0000201254 \\
163840 & 0.001383 & 0.0000100629 \\
327680 & 0.001380 & 0.0000050315 \\
655360 & 0.001372 & 0.000002516 \\
1310720 & 0.001398 & 0.000001258 \\
\hline
\end{tabular}

at the point $s=0.5$ respectively for the function $e^{x}$ and $\sin (\log x)$. We end the tabulations when the values start becoming worse, because of the numerical approximation problems.

Finally we show that the Hadamard integrals for the function $e^{x}$ do not converge uniformly on $] 0, \infty)$. To this end, we evaluate in Table 3 the difference $\left|\left(J_{0^{+}, w}^{2}\right)(w)-e^{w}\right|$ as $w \rightarrow \infty$ (as far as it is numerically possible to go!): In this case, the trapezoidal rule has been splitted into two parts: one for the interval $[0,1]$ and the other for the interval $[1, w]$; the number of points taken for both 
Table 2. Hadamard integrals values for $f=\sin (\log x)$ on $s=0.5$.

\begin{tabular}{rll}
\hline$w$ & $\sin (\log 0.5)-\left(J_{0^{+}, w}^{2} f\right)(0.5)$ & $\begin{array}{l}\text { Gauss-Legendre quadrature rule } \\
\text { with } 8192 \text { points }\end{array}$ \\
\hline 10 & 0.131360 & 0.1319625976 \\
20 & 0.071231 & 0.0717683965 \\
40 & 0.036687 & 0.0372171072 \\
80 & 0.018394 & 0.0189255291 \\
160 & 0.009008 & 0.0095398617 \\
320 & 0.004256 & 0.0047889300 \\
640 & 0.001866 & 0.0023991800 \\
1280 & 0.000668 & 0.0012007643 \\
2560 & 0.000068 & 0.0006006752 \\
5120 & -0.000231 & 0.0003004108 \\
\hline
\end{tabular}

Table 3.

\begin{tabular}{rll}
\hline$w$ & $e^{w}-\left(J_{0^{+}, w}^{2} e^{x}\right)(w)$ & $\begin{array}{l}\text { Gauss-Legendre quadrature rule } \\
\text { with 2048 points }\end{array}$ \\
\hline 10 & 16082.6708556401 & 16082.5451 \\
20 & 359195544.652526 & 359191896 \\
40 & $1.75421291539295 e+17$ & $1.75419427 e+17$ \\
80 & $4.1424310899933 e+34$ & $4.14238607 e+34$ \\
160 & $2.29880188317864 e+69$ & $2.29877662 e+69$ \\
320 & $7.06252872079612 e+138$ & $7.06245068 e+138$ \\
640 & $6.65831993738322 e+277$ & $6.65824616 e+277$ \\
\hline
\end{tabular}

Table 4.

\begin{tabular}{rll}
\hline $\mathrm{w}$ & $e^{640}-\left(J_{0^{+}, w}^{2} e^{x}\right)(640)$ & $\begin{array}{l}\text { Gauss-Legendre quadrature rule } \\
\text { with } 4096 \text { points }\end{array}$ \\
\hline 640 & $6.665632367 e+277$ & $6.6582462 e+277$ \\
1280 & $4.939299747 e+277$ & $4.9319071 e+277$ \\
2560 & $3.203537754 e+277$ & $3.1961426 e+277$ \\
5120 & $1.870931917 e+277$ & $1.8635357 e+277$ \\
10240 & $1.021377889 e+277$ & $1.0139811 e+277$ \\
20480 & $0.537456303 e+277$ & $0.5300593 e+277$ \\
40960 & $0.278551328 e+277$ & $0.2711541 e+277$ \\
81920 & $0.144552914 e+277$ & $0.1371559 e+277$ \\
163840 & $0.076373845 e+277$ & $0.0689788 e+277$ \\
\hline
\end{tabular}

the numerical integrations is $100 * w$.

For the sake of completeness, we report also the values showing the (slow) pointwise convergence in $s=640$ (see Table 4 ).

\section{Acknowledgments}

The authors express their gratitude to the Referees for their valuable suggestions which greatly improved the presentation of the paper. In particular 
we wish to thank the Referee who suggested us a Gauss-Legendre quadrature rule for the evaluation of the integrals. The authors have been partially supported by the Gruppo Nazionale Analisi Matematica, Probabilità e Applicazioni (GNAMPA) of the Istituto Nazionale di Alta Matematica (INdAM), through the INdAM-GNAMPA Project 2014, and by the Department of Mathematics and Computer Science of University of Perugia.

\section{References}

[1] G.A. Anastassiou and S.G. Gal. Approximation Theory. Moduli of Continuity and Global Smoothness Preservation. Birkhäuser Boston, Inc., Boston, 2000.

[2] L. Angeloni. A characterization of a modulus of smoothness in multidimensional setting. Boll. Unione Mat. Ital., 4(1):79-108, 2011.

[3] L. Angeloni and G. Vinti. Approximation in variation by homothetic operators in multidimensional setting. Differential Integral Equations, 26(5-6):655-674, 2013.

[4] F. Barbieri. Approximation by moment kernels. Atti Sem. Mat. Fis. Univ. Modena, 32(2):308-328, 1983.

[5] C. Bardaro, P.L. Butzer and I. Mantellini. The foundations of the fractional calculus in Mellin transform setting with applications. J. Fourier Anal. Appl., 2015. To appear

[6] C. Bardaro and I. Mantellini. Linear integral operators with homogeneous kernel: approximation properties in modular spaces. Applications to Mellin-type convolution operators and to some classes of fractional operators. In G.A. Anastassiou(Ed.), Applied Mathematics Reviews, volume 1, pp. 45-67. World Scientific Publ., River Edge, NJ, 2000. http://dx.doi.org/10.1142/9789812792686_0003.

[7] C. Bardaro and I. Mantellini. Voronovskaya-type estimates for Mellin convolution operators. Results Math., 50(1-2):1-16, 2007. http://dx.doi.org/10.1007/s00025-006-0231-3.

[8] C. Bardaro and I. Mantellini. A quantitative Voronovskaja formula for Mellin convolution operators. Mediterr. J. Math., 7(4):483-501, 2010. http://dx.doi.org/10.1007/s00009-010-0062-z.

[9] C. Bardaro and I. Mantellini. Approximation properties for linear combinations of moment type operators. Comput. Math. Appl., 62:2304-2313, 2011. http://dx.doi.org/10.1016/j.camwa.2011.07.017.

[10] C. Bardaro and I. Mantellini. A note on the Voronovskaja theorem for MellinFejer convolution operators. Appl. Math. Lett., 24:2064-2067, 2011. http://dx.doi.org/10.1016/j.aml.2011.05.043.

[11] C. Bardaro and I. Mantellini. On the iterates of Mellin-Fejer convolution operators. Acta Appl. Math., 121:213-229, 2012. http://dx.doi.org/10.1007/s10440-012-9704-4.

[12] C. Bardaro and I. Mantellini. On Mellin convolution operators: a direct approach to the asymptotic formulae. Integral Transforms Spec. Funct., 25(3):182-195, 2014. http://dx.doi.org/10.1080/10652469.2013.838755.

[13] C. Bardaro and G. Vinti. Modular convergence in generalized Orlicz spaces for moment type operators. Appl. Anal., 32:265-276, 1989. http://dx.doi.org/10.1080/00036818908839853. 
[14] A. Boccuto, D. Candeloro and A. Sambucini. Vitali-type theorems for filter convergence related to Riesz space-valued modulars and applications to stochastic processes. J. Math. Anal. Appl., 419(2):818-838, 2014. http://dx.doi.org/10.1016/j.jmaa.2014.05.014.

[15] P.L. Butzer and H. Berens. Semi-Groups of Operators and Approximation. Springer-Verlag, Berlin, Heidelberg, New York, 1967.

[16] P.L. Butzer and S. Jansche. A direct approach to the Mellin transform. J. Fourier Anal. Appl., 3:325-375, 1997. http://dx.doi.org/10.1007/BF02649101.

[17] P.L. Butzer, A.A. Kilbas and J.J. Trujillo. Fractional calculus in the Mellin setting and Hadamard-type fractional integral. J. Math. Anal. Appl., 269:1-27, 2002. http://dx.doi.org/10.1016/S0022-247X(02)00001-X.

[18] D. Candeloro and A.R. Sambucini. Filter convergence and decompositions for vector lattice-valued measures. Mediterr. J. Math., 2014. http://dx.doi.org/10.1007/s00009-014-0431-0.

[19] R.A. DeVore and G.G. Lorentz. Constructive Approximation. Springer-Verlag, Berlin, Heidelberg, 1993.

[20] C. Fiocchi. Variazione di ordine $\alpha$ e dimensione di Hausdorff degli insiemi di Cantor. Atti Sem. Mat. Fis. Univ. Modena, 34(2):649-667, 1991.

[21] H. Gonska, P. Pitul and I. Rasa. On Peano's form of the Taylor remainder, Voronovskaja's theorem and the commutator of positive linear operators. In O. Agratini and P. Blaga(Eds.), Inter. Conf. Numerical Analysis and Approximation Theory, pp. 55-80, Cluj-Napoca, Romania, 2006.

[22] J. Hadamard. Essai sur l'etude des fonctions donnees par leur developpement de Taylor. J. Math. Pures Appl., 8:101-186, 1892.

[23] A.A. Kilbas, H.M. Srivastava and J.J. Trujillo. Theory and Applications of Fractional Differential Equations. Elsevier, Amsterdam, 2006.

[24] L. Maligranda. Interpolation spaces in the theory of approximation. In Methods of Functional Analysis in Approximation Theory, volume 2, pp. 263-279, ISNM 76, Bombay, 1985, 1986. Birkhäuser.

[25] R.G. Mamedov. The Mellin Transform and Approximation Theory. Elm, Baku, 1991. (in Russian)

[26] J. Peetre. Exact interpolation theorems for Lipschitz continuous functions. Ricerche Mat., 18:239-259, 1969.

[27] F. Ventriglia and G. Vinti. Nonlinear Kantorovich-type operators: a unified approach. Comm. Appl. Nonlinear Anal., 21(2):45-74, 2014.

[28] C. Vinti. Sull'approssimazione in perimetro e in area. Atti Sem. Mat. Fis. Univ. Modena, 13:187-197, 1964.

[29] G. Vinti. A general approximation result for nonlinear integral operators and applications to signal processing. Appl. Anal., 79:217-238, 2001. http://dx.doi.org/10.1080/00036810108840958.

[30] G. Vinti and L. Zampogni. A unifying approach to convergence of linear sampling type operators in Orlicz spaces. Adv. Differential Equations, 16:573-600, 2011.

[31] V. Zanelli. Funzioni momento convergenti dal basso in variazione di ordine non intero. Atti Sem. Mat. Fis. Univ. Modena, 30:355-369, 1981. 